\title{
Impact of modified
}

albumin-bilirubin grade on survival in patients with HCC who received lenvatinib

\author{
Toshifumi Tada $\mathbb{1}^{1 \bowtie}$, Takashi Kumada2 ${ }^{2}$, Atsushi Hiraoka ${ }^{3}$, Masanori Atsukawa ${ }^{4}$, \\ Masashi Hirooka ${ }^{5}$, Kunihiko Tsuji ${ }^{6}$, Toru Ishikawa ${ }^{7}, K^{2}$ ichi Takaguchi ${ }^{8}$, Kazuya Kariyama ${ }^{9}$, \\ Ei Itobayashi ${ }^{10}$, Kazuto Tajiri ${ }^{11}$, Noritomo Shimada ${ }^{12}$, Hiroshi Shibata ${ }^{13}$, Hironori Ochi ${ }^{14}$, \\ Satoshi Yasuda ${ }^{15}$, Hidenori Toyoda ${ }^{15}$, Shinya Fukunishi ${ }^{16}$, Hideko Ohama ${ }^{16}$, \\ Kazuhito Kawata ${ }^{17}$, Joji Tani ${ }^{18}$, Shinichiro Nakamura ${ }^{1}$, Kazuhiro Nouso ${ }^{9}$, Akemi Tsutsui ${ }^{8}$, \\ Takuya Nagano ${ }^{8}$, Tanaka Takaaki ${ }^{3}$, Norio Itokawa ${ }^{4}$, Tomomi Okubo ${ }^{4}$, Taeang Arai ${ }^{4}$, \\ Michitaka Imai ${ }^{7}$, Kouji Joko ${ }^{14}$, Yohei Koizumi ${ }^{5}$, Yoichi Hiasa ${ }^{5}$ \& Real-life Practice Experts for \\ HCC (RELPEC) Study Group and the HCC 48 Group (hepatocellular carcinoma experts from 48 \\ clinics in Japan)
}

We investigated the impact on survival of modified albumin-bilirubin (mALBI) grade versus ChildPugh classification in patients with hepatocellular carcinoma (HCC) who received lenvatinib. A total of 524 patients with $\mathrm{HCC}$ who received lenvatinib were included. Univariate analysis showed that mALBI grade $2 \mathrm{~b} / 3$ and Child-Pugh class $B / C$ were significantly associated with survival [hazard ratio (HR), 2.471; 95\% confidence interval (Cl), 1.944-3.141 and $\mathrm{HR}, 2.178 ; 95 \% \mathrm{Cl}, 1.591-2.982$ ]. In patients with a Child-Pugh score of 5 , multivariate analysis showed that mALBI grade $2 \mathrm{~b} / 3$ was independently associated with survival $(\mathrm{HR}, 1.814 ; 95 \% \mathrm{Cl}, 1.083-3.037)$. Conversely, among patients with $\mathrm{mALBI}$ grade 1/2a, there was no difference in survival between those with a Child-Pugh class of 5 or $6(p=0.735)$. Time-dependent receiver operating characteristic analysis showed that the ALBI score predicted survival better than the Child-Pugh score. The optimal cut-off value of the ALBI score for predicting survival was nearly the same as the value separating mALBI grades $2 \mathrm{a}$ and $2 \mathrm{~b}$. In conclusion, the MALBI grade was a better predictor of survival than the Child-Pugh classification in patients with unresectable HCC who received lenvatinib therapy.

Hepatocellular carcinoma (HCC) accounts for the majority of malignancy in the liver. It is also one of the most prevalent cancers worldwide and the cause of important health-related problems, making it the third

\footnotetext{
${ }^{1}$ Department of Internal Medicine, Japanese Red Cross Himeji Hospital, 1-12-1 Shimoteno, Himeji, Hyogo 670-8540, Japan. ${ }^{2}$ Faculty of Nursing, Gifu Kyoritsu University, Ogaki, Japan. ${ }^{3}$ Gastroenterology Center, Ehime Prefectural Central Hospital, Matsuyama, Japan. ${ }^{4}$ Division of Gastroenterology and Hepatology, Department of Internal Medicine, Nippon Medical School, Tokyo, Japan. ${ }^{5}$ Department of Gastroenterology and Metabology, Ehime University Graduate School of Medicine, Matsuyama, Japan. ${ }^{6}$ Center of Gastroenterology, Teine Keijinkai Hospital, Sapporo, Japan. ${ }^{7}$ Department of Gastroenterology, Saiseikai Niigata Hospital, Niigata, Japan. ${ }^{8}$ Department of Hepatology, Kagawa Prefectural Central Hospital, Takamatsu, Japan. ${ }^{9}$ Department of Gastroenterology, Okayama City Hospital, Okayama, Japan. ${ }^{10}$ Department of Gastroenterology, Asahi General Hospital, Asahi, Japan. ${ }^{11}$ Department of Gastroenterology, Toyama University Hospital, Toyama, Japan. ${ }^{12}$ Division of Gastroenterology and Hepatology, Otakanomori Hospital, Kashiwa, Japan. ${ }^{13}$ Department of Gastroenterology, Tokushima Prefectural Central Hospital, Tokushima, Japan. ${ }^{14}$ Hepato-Biliary Center, Matsuyama Red Cross Hospital, Matsuyama, Japan. ${ }^{15}$ Department of Gastroenterology and Hepatology, Ogaki Municipal Hospital, Ogaki, Japan. ${ }^{16}$ Second Department of Internal Medicine, Osaka Medical College, Takatsuki, Japan. ${ }^{17}$ Hepatology Division, Department of Internal Medicine, Hamamatsu University School of Medicine, Hamamatsu, Japan. ${ }^{18}$ Department of Gastroenterology and Neurology, Kagawa University School of Medicine, Miki, Kagawa, Japan. ${ }^{\square}$ email: tadat0627@gmail.com
} 


\begin{tabular}{|c|c|c|c|}
\hline & Overall $(n=524)$ & Child-Pugh A $(n=448)$ & Child-Pugh score of $5(n=277)$ \\
\hline Age (years)* & $73.0(68.0-79.0)$ & $73.0(68.0-79.0)$ & $73.0(68.0-79.0)$ \\
\hline Sex (female/male) & $126 / 398$ & $106 / 342$ & $59 / 218$ \\
\hline ECOG-PS $(0 / 1 / 2 / 3)$ & $420 / 91 / 12 / 1$ & $367 / 72 / 8 / 1$ & $233 / 40 / 3 / 1$ \\
\hline Body mass index $\left(\mathrm{kg} / \mathrm{m}^{2}\right)^{\star}$ & $22.9(20.6-25.4)$ & $22.9(20.8-25.5)$ & $23.1(20.9-25.4)$ \\
\hline $\begin{array}{l}\text { Etiology of HCC (hepatitis } B / C / B+C / \text { non-B, } \\
\text { non-C) }\end{array}$ & $75 / 214 / 2 / 233$ & 69/179/2/198 & $49 / 105 / 2 / 121$ \\
\hline Albumin $(\mathrm{g} / \mathrm{dL})^{*}$ & $3.6(3.3-4.0)$ & $3.8(3.4-4.0)$ & $3.9(3.7-4.2)$ \\
\hline Total bilirubin $(\mathrm{mg} / \mathrm{dL})^{*}$ & $0.8(0.6-1.1)$ & $0.7(0.6-1.0)$ & $0.7(0.6-1.0)$ \\
\hline Platelet count $\left(\times 10^{3} / \mathrm{m}^{3}\right)^{*}$ & $13.5(10.0-18.6)$ & $13.5(10.2-18.1)$ & $14.3(10.8-18.5)$ \\
\hline Prothrombin time $(\%)^{*}$ & $87.0(78.0-97.0)$ & $88.3(80.0-98.0)$ & $90.0(83.0-100.0)$ \\
\hline$\alpha$-fetoprotein $(\mathrm{ng} / \mathrm{mL})^{*}$ & $40.9(6.8-668.7)$ & $35.0(6.1-497.2)$ & $25.7(5.6-426.0)$ \\
\hline ALBI score* & $-2.36(-2.68$ to -1.99$)$ & $-2.47(-2.73$ to -2.17$)$ & $-2.65(-2.86$ to -2.44$)$ \\
\hline mALBI grade $(1 / 2 \mathrm{a} / 2 \mathrm{~b} / 3)$ & $165 / 131 / 210 / 18$ & $165 / 130 / 152 / 1$ & $153 / 95 / 28 / 1$ \\
\hline Child-Pugh score (5/6/7/8/9/10/13) & $277 / 171 / 56 / 15 / 2 / 2 / 1$ & $277 / 171$ & \\
\hline Child-Pugh class (A/B/C) & $448 / 73 / 3$ & & \\
\hline BCLC stage $(0 / \mathrm{A} / \mathrm{B} / \mathrm{C} / \mathrm{D})$ & $4 / 8 / 225 / 285 / 2$ & $4 / 8 / 198 / 237 / 1$ & $3 / 7 / 126 / 140 / 1$ \\
\hline Vascular invasion (yes/no) & $116 / 408$ & $95 / 353$ & $51 / 226$ \\
\hline Extrahepatic spread (yes/no) & $186 / 338$ & $158 / 290$ & $96 / 181$ \\
\hline Molecular targeted therapy experience (yes/no) & $155 / 369$ & $133 / 315$ & $79 / 198$ \\
\hline Line of lenvatinib (1st/2nd/3rd/4th) & $369 / 110 / 44 / 1$ & $315 / 95 / 37 / 1$ & $198 / 59 / 20 / 0$ \\
\hline Post-treatment of lenvatinib (yes/no/ongoing) & $188 / 253 / 83$ & $169 / 204 / 75$ & $125 / 101 / 51$ \\
\hline Follow-up duration (months) & $11.6(6.0-18.5)$ & $12.4(7.0-19.2)$ & $14.3(10.8-18.5)$ \\
\hline
\end{tabular}

Table 1. Patient characteristics. ${ }^{\star}$ Values are expressed as medians (interquartile range). ECOG-PS Eastern Cooperative Oncology Group performance status, HCC Hepatocellular carcinoma, ALBI Albumin-bilirubin, $m A L B I$ Modified albumin-bilirubin, $B C L C$ Barcelona clinic liver cancer.

most frequent cause of cancer-related deaths in the world ${ }^{1-3}$. Curative treatments such as hepatic resection, radiofrequency/microwave ablation, and transplantation are performed in only $30-40 \%$ of patients with HCC. Remaining patients for whom curative treatment is not indicated undergo transarterial chemoembolization or systemic drug therapy for palliation ${ }^{4}$.

Sorafenib ${ }^{5,6}$, regorafenib ${ }^{7,8}$, and ramucirumab ${ }^{9}$ are attributed as molecularly targeted agents and have been developed for the systemic drug therapy of patients with unresectable HCC. In the past 10 years, no first-line systemic drug therapies other than sorafenib were approved for patients with unresectable HCC in Japan ${ }^{5-8}$. Lenvatinib $^{10}$, a newly developed tyrosine kinase inhibitor, has recently become available not only as a first-line therapy in patients with unresectable HCC, but also as a later-line therapeutic option ${ }^{11,12}$. More recently in Japan, the combination of atezolizumab plus bevacizumab has been approved as a first-line systemic therapy for patients with unresectable $\mathrm{HCC}^{13}$.

The Child-Pugh classification system ${ }^{14}$ is the most extensively and globally used method for evaluating hepatic function in patients being treated for HCC. However, this system includes subjective factors such as hepatic encephalopathy and ascites, and interrelated factors such as serum albumin and ascites. Therefore, accurate and more objective clinical markers to evaluate hepatic function are desired. A new method for assessing hepatic function, known as the albumin-bilirubin (ALBI) grade, was recently developed ${ }^{15}$. In addition, several studies have shown that the modified ALBI (mALBI) grade is effective for assessing hepatic function in patients with $\mathrm{HCC}^{16-18}$. Furthermore, we recently reported that hepatic function in patients with naïve HCC has improved remarkably throughout 30 years, and in the last 5 years over $80 \%$ of patients reached Child-Pugh class A, including $65 \%$ who attained a Child-Pugh score of $5^{19}$. In addition, we recently reported that Child-Pugh score of 6 was independently associated with overall survival in HCC patients with Child-Pugh class A who were treated with sorafenib [hazard ratio (HR), 1.41; 95\% confidence interval (CI), 1.08-1.84] $]^{20}$. Since lenvatinib therapy is mainly indicated in HCC patients with Child-Pugh class A disease, it is necessary to elucidate the ability of the mALBI grade to assess hepatic function as an alternative to the Child-Pugh classification system.

In this study, we investigated the association between the ALBI score and overall survival, with a particular focus on the mALBI grade versus the Child-Pugh score, in patients with unresectable HCC who treated with lenvatinib at multiple centers in Japan. To further compare the ability of the ALBI score and Child-Pugh score in predicting overall survival, we generated time-dependent receiver operating characteristic (ROC) curves analysis ${ }^{21}$ for censored data and compared the areas under the ROC curves (AUROCs).

\section{Results}

Patient characteristics. Table 1 shows the characteristics of the study patients at the baseline. There were $126(24.0 \%)$ females and $398(76.0 \%)$ males, with a median age of 73.0 (68.0-79.0) years. The median follow-up period was $11.6(6.0-18.5)$ months. There were 277 (52.9\%) patients with a Child-Pugh score of 5, $171(32.6 \%)$ with a score of $6,56(10.7 \%)$ with a score of $7,15(2.9 \%)$ with a score of $8,2(0.4 . \%)$ with a score of $9,2(0.4 \%)$ 
with a score of 10 , and $1(0.2 \%)$ with a score of 13 , corresponding to 448 (85.5\%) patients with Child-Pugh class A disease, $73(13.9 \%)$ with class B disease, and $3(0.6 \%)$ patients with class C disease. The median ALBI score was $-2.36(-2.68$ to -1.99$)$. There were $165(31.5 \%)$ patients with mALBI grade $1,131(25.0 \%)$ with grade $2 \mathrm{a}$, $210(40.1 \%)$ with grade $2 \mathrm{~b}$, and $18(3.4 \%)$ with grade 3 .

The cumulative overall survival rates at $6,12,18$, and 24 months were $82.7 \%, 61.6 \%, 47.3 \%$, and $37.0 \%$, respectively. The median overall survival was 17.1 months (95\% CI, 15.0-19.6). There were 45 (27.3\%), 44 (33.6\%), $59(28.1 \%)$, and $7(38.9 \%)$ patients with mALBI grade $1 / 2 \mathrm{a} / 2 \mathrm{~b} / 3$ who had an experience of molecular targeted therapy $(p=0.493)$. Additionally, there were $133(29.7 \%), 21(28.8 \%)$, and $1(33.3 \%)$ patients with Child-Pugh class $\mathrm{A} / \mathrm{B} / \mathrm{C}$ who had an experience of molecular targeted therapy $(p=0.977)$.

Overall survival by mALBI grade and Child-Pugh class. Figure 1a shows that cumulative overall survival curves differed significantly according to mALBI grade $(p<0.001)$. Multiple comparisons using the Bonferroni method showed significant differences between each grade, except between mALBI grades 1 and $2 \mathrm{a}$, and grades $2 \mathrm{~b}$ and 3 . Figure $1 \mathrm{~b}$ shows a significant difference between the cumulative overall survival curves of patients with mALBI grades of $1 / 2$ a versus $2 \mathrm{~b} / 3(p<0.001)$. Univariate Cox proportional hazards model analysis showed that mALBI grade $2 \mathrm{~b} / 3$ was significantly associated with poor overall survival (HR, 2.471 ; $95 \% \mathrm{CI}$, $1.944-3.141 ; p<0.001$; c-index, 0.625). Multivariate Cox proportional hazards models analysis that included the covariates of age, sex, ECOG-PS, etiology, $\alpha$-fetoprotein, HCC stage, and mALBI grade showed that HCC stage $(\mathrm{HR}, 1.412)$ and $\mathrm{mALBI}$ grade $(\mathrm{HR}, 2.399)$ were independently associated with overall survival (Supplementary Table 1).

Figure 1c shows that cumulative overall survival curves differed significantly according to Child-Pugh class $(p<0.001)$. Multiple comparisons using the Bonferroni method showed a significant difference only between Child-Pugh classes A and B. Figure 1d shows a significant difference between cumulative overall survival curves for Child-Pugh class A versus B/C $(p<0.001)$. Univariate Cox proportional hazards model analysis showed that Child-Pugh class B/C was significantly associated with poor overall survival (HR, 2.178; 95\% CI, 1.591-2.982; $p<0.001$; c-index, 0.555). Multivariate Cox proportional hazards models analysis that included the covariates of age, sex, ECOG-PS, etiology, a-fetoprotein, HCC stage, and Child-Pugh class showed that HCC stage (HR, 1.402) and Child-Pugh class (HR, 2.048) were independently associated with overall survival (Supplementary Table 2).

Therapeutic response. Radiological best response rates according to the mALBI grade and Child-Pugh class show supplementary Tables 3 and 4, respectively. Overall response rate (ORR) and disease control rate (DCR) in patients with mALBI grade $1 / 2 \mathrm{a}$ and $2 \mathrm{~b} / 3$ were $40.8 \%$ and $83.0 \%$, and $32.3 \%$ and $75.3 \%$, respectively. DCR was significantly difference between the patients with mALBI grade $1 / 2 \mathrm{a}$ and $2 \mathrm{~b} / 3(p=0.049)$ (Supplementary Table 3). In addition, ORR and DCR in patients with Child-Pugh class A and B/C were $38.6 \%$ and $82.6 \%$, and $28.8 \%$ and $62.1 \%$, respectively. DCR was significantly difference between the patients with Child-Pugh class $\mathrm{A}$ and $\mathrm{B} / \mathrm{C}(p<0.001)$ (Supplementary Table 4$)$.

Adverse events. Supplementary Tables 5 and 6 lists the treatment-related adverse events that occurred in study patients according to the mALBI grade and Child-Pugh class. Regarding to the relationship between adverse events, liver function, and overall survival, multivariate Cox proportional hazards models analysis that included the covariates of adverse events and mALBI grade showed that palmar-plantar erythrodysesthesia (HR, $0.679)$, proteinuria ( $\mathrm{HR}, 0.646)$, and $\mathrm{mALBI}$ grade ( $\mathrm{HR}, 2.234)$ were independently associated with overall survival (Supplementary Table 7). In addition, multivariate Cox proportional hazards models analysis that included the covariates of adverse events and Child-Pugh class showed that palmar-plantar erythrodysesthesia (HR, $0.648)$, proteinuria (HR, 0.584), and Child-Pugh class (HR, 2.021) were independently associated with overall survival (Supplementary Table 8).

Subgroup analysis. Table 1 shows the characteristics of patients with a Child-Pugh score of 5 at the start of follow-up $(\mathrm{n}=277)$. There were 59 females and 218 males, with a median age of $73.0(68.0-79.0)$ years. There were $153(55.2 \%)$ patients with mALBI grade $1,95(34.3 \%)$ with grade $2 \mathrm{a}, 28(10.1 \%)$ with grade $2 \mathrm{~b}$, and $1(0.4 \%)$ with grade 3 . Figure 2 shows a significant difference between cumulative overall survival curves for mALBI grade $1 / 2$ a versus $2 \mathrm{~b} / 3(p=0.032)$. Univariate Cox proportional hazards model analysis showed that mALBI grade $2 \mathrm{~b} / 3$ was significantly associated with poor overall survival (HR, 1.740; 95\%CI, 1.040-2.909; $p=0.035$ ). Multivariate Cox proportional hazards models analysis that included the covariates of age, sex, ECOG-PS, etiology, $a$-fetoprotein, HCC stage, and mALBI grade showed that mALBI grade was independently associated with overall survival (HR, 1.814; 95\%CI, 1.083-3.037; $p=0.024)($ Table 2$)$. There were $116(58.0 \%)$ and 84 $(42.0 \%)$ patients with $\mathrm{mALBI}$ grade $1 / 2 \mathrm{a}$ who received post-treatment of lenvatinib and not, and $9(34.6 \%)$ and $17(65.4 \%)$ patients with mALBI grade $2 \mathrm{~b} / 3$ who received post-treatment of it and not, respectively $(p=0.035)$.

Figure 3 shows that in patients with $\mathrm{mALBI}$ grade $1 / 2 \mathrm{a}(\mathrm{n}=295)$, there was no significant difference in cumulative overall survival curves according to Child-Pugh scores of 5 versus $6(p=0.735)$. Univariate Cox proportional hazards model analysis showed that a Child-Pugh class of 6 was not associated with poor overall survival (HR, 1.088; 95\%CI, 0.666-1.777; $p=0.736)$.

Time-dependent ROC analysis of overall survival. Supplementary Fig. 1a-h shows the ROC curves of ALBI scores for overall survival at 3, 6, 9, 12, 15, 18, 21, and 24 months, respectively, after the start of follow-up using time-dependent ROC analysis. The AUCs at 3, 6, 9, 12, 15, 18, 21, and 24 months were 0.682, 0.693, 0.673, $0.640,0.641,0.621,0.615$, and 0.621 , respectively. Supplementary Fig. 2a-h shows the ROC curves of ChildPugh scores for overall survival at 3, 6, 9, 12, 15, 18, 21, and 24 months, respectively, after the start of follow-up 

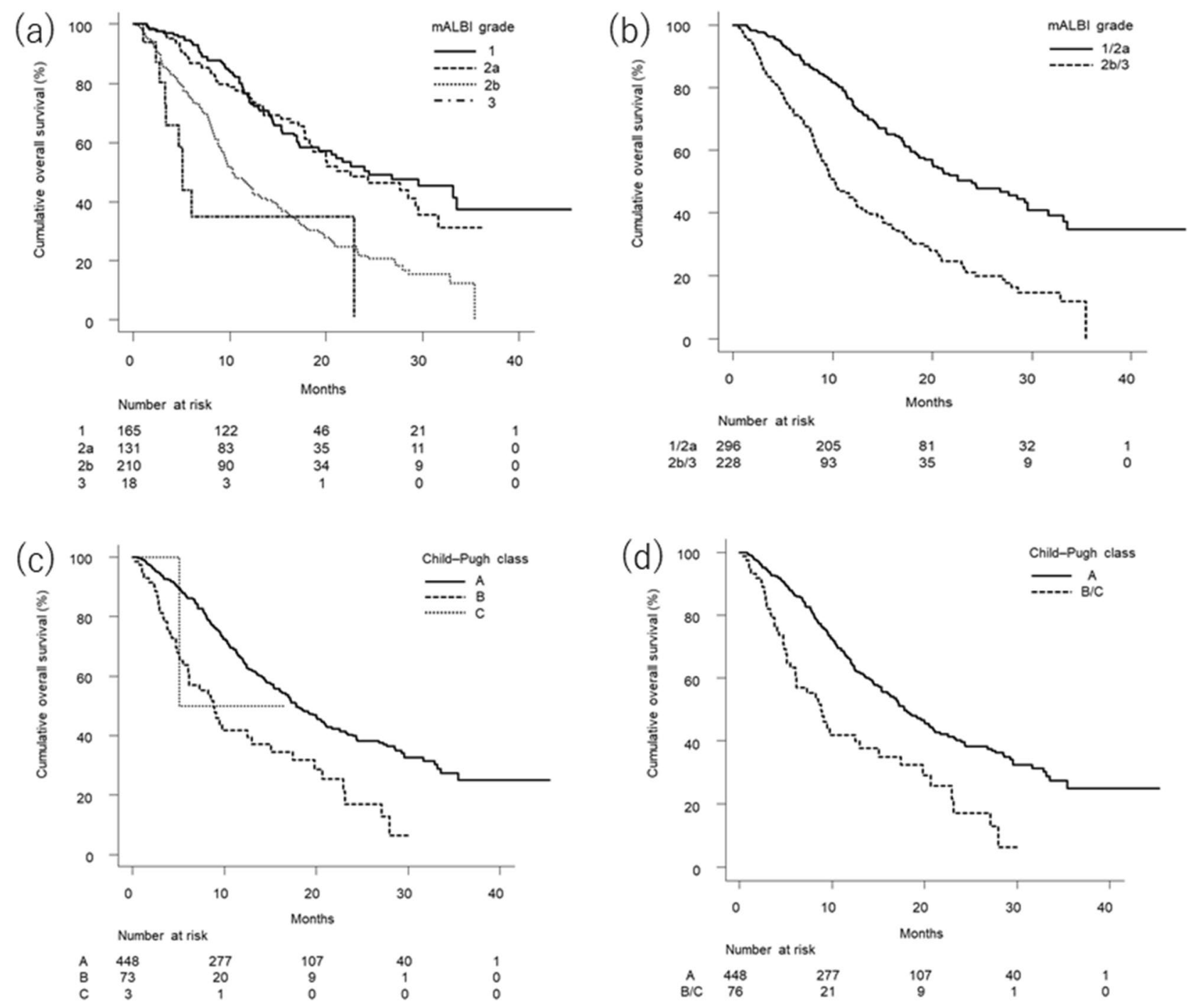

Figure 1. Cumulative survival curves for all study patients. (a) By mALBI grade. There was a significant difference in cumulative overall survival among patients grouped by mALBI grade $(p<0.001)$. The median survival times of patients with mALBI grades 1, 2a, 2b, and 3 were 24.4 (95\% CI, 17.2—not available), 22.5 (95\%CI, 18.3-29.5), 10.3 (95\%CI, 9.1-12.5), and 5.1 (95\%CI, 3.3-not available) months, respectively. Multiple comparisons between mALBI grades 1 and $2 \mathrm{a}, 1$ and $2 \mathrm{~b}, 1$ and $3,2 \mathrm{a}$ and $2 \mathrm{~b}, 2 \mathrm{a}$ and 3 , and $2 \mathrm{~b}$ and 3 demonstrated p values of $1.000,<0.001,<0.001,<0.001,<0.001$, and 0.654 , respectively. (b) By mALBI grade divided into $1 / 2 \mathrm{a}$ and $2 \mathrm{~b} / 3$. There was a significant difference in cumulative overall survival between patients with mALBI grades $1 / 2 \mathrm{a}$ and $2 \mathrm{~b} / 3(p<0.001)$. The median survival times in patients with mALBI grades $1 / 2 \mathrm{a}$ and $2 \mathrm{~b} / 3$ were 23.9 (95\%CI, 20.0-29.5) and 10.1 (95\%CI, 8.9-12.4) months, respectively. (c) By Child-Pugh classification. There was a significant difference in cumulative overall survival among patients stratified by Child-Pugh classification $(p<0.001)$. The median survival times in patients with Child-Pugh classes A, B, and C were 17.8 (95\%CI, 16.0-20.8), 8.8 (95\%CI, 6.0-12.9), and 5.1 (95\%CI, 5.1-not available) months, respectively. Multiple comparisons between Child-Pugh classes A and B, A and C, and B and C demonstrated $\mathrm{p}$ values of $<0.001,1.000$, and 1.000, respectively. (d) By Child-Pugh classification divided into A and B/C. There was a significant difference in cumulative overall survival between patients with Child-Pugh classes $\mathrm{A}$ and $\mathrm{B} / \mathrm{C}(p<0.001)$. The median survival times in patients with Child-Pugh classes A and B/C were 17.8 (95\%CI, 16.0-20.8) and 8.8 (95\%CI, 6.0-15.0) months, respectively. mALBI Modified albumin-bilirubin, CI Confidence interval.

using time-dependent ROC analysis. The AUCs at 3, 6, 9, 12, 15, 18, 21, and 24 months were 0.738, 0.719, 0.712, $0.673,0.663,0.658,0.664$, and 0.671 , respectively.

Figure 4 shows AUROC plots of the ALBI and Child-Pugh scores for overall survival from 3 to 24 months after the start of follow-up, as analyzed by time-dependent ROC. The ALBI score had better predictive ability for overall survival than the Child-Pugh score at all time points.

Table 3 shows the sensitivity, specificity, and cut-off values of the ALBI and Child-Pugh scores at 6, 12, 18, and 24 months according to time-dependent ROC analysis for overall survival. The optimal cut-off values of the ALBI 


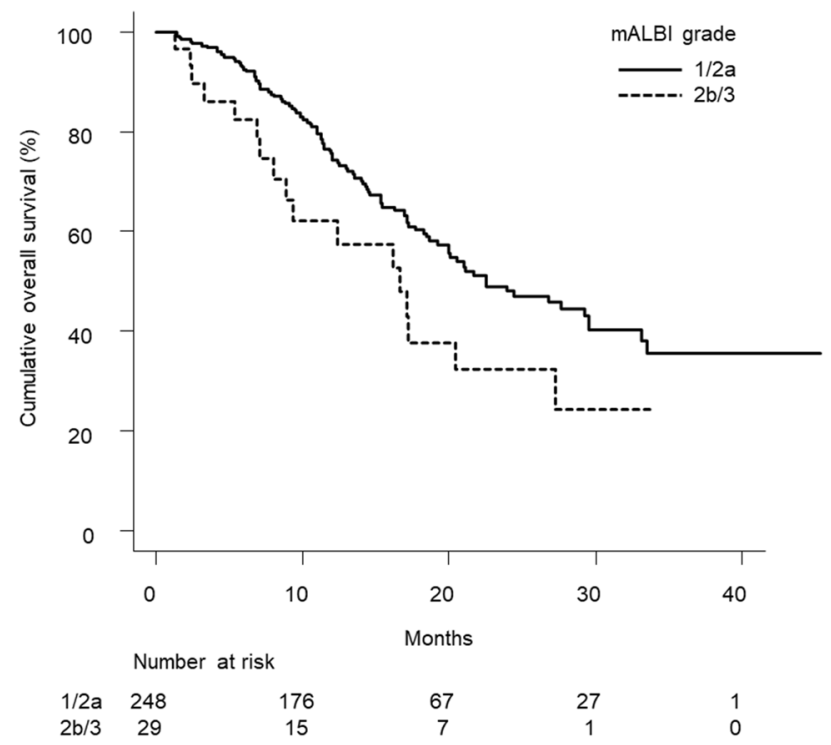

Figure 2. Cumulative survival curves in patients with Child-Pugh score of 5 . There was a significant difference in cumulative overall survival between patients with mALBI grades $1 / 2 \mathrm{a}$ and $2 \mathrm{~b} / 3(p=0.032)$. The median survival times in patients with mALBI grades $1 / 2 \mathrm{a}$ and $2 \mathrm{~b} / 3$ were $22.5(95 \% \mathrm{CI}, 19.2-29.5)$ and 16.6 (95\%CI, 8.0-27.3) months, respectively.

\begin{tabular}{|c|c|c|c|}
\hline & HR & $95 \%$ CI & $p$ value \\
\hline \multicolumn{4}{|l|}{ Age (years) } \\
\hline$<75(\mathrm{n}=161)$ & 1 & $0.899-1.945$ & 0.155 \\
\hline$\geq 75(\mathrm{n}=116)$ & 1.322 & & \\
\hline \multicolumn{4}{|l|}{ Sex } \\
\hline Female $(n=59)$ & 1 & $0.675-1.680$ & 0.786 \\
\hline Male $(n=218)$ & 1.065 & & \\
\hline \multicolumn{4}{|l|}{ ECOG-PS } \\
\hline $0(n=233)$ & 1 & $0.506-1.562$ & 0.682 \\
\hline$\geq 1(\mathrm{n}=44)$ & 0.889 & & \\
\hline \multicolumn{4}{|l|}{ Etiology of HCC } \\
\hline Viral $(n=156)$ & 1 & \begin{tabular}{|l|}
$0.497-1.079$ \\
\end{tabular} & 0.115 \\
\hline Non-viral $(\mathrm{n}=121)$ & 0.732 & & \\
\hline \multicolumn{4}{|c|}{ a-fetoprotein $(\mathrm{ng} / \mathrm{mL})$} \\
\hline$<400(\mathrm{n}=205)$ & 1 & $0.938-2.120$ & 0.099 \\
\hline$\geq 400(\mathrm{n}=71)$ & 1.410 & & \\
\hline \multicolumn{4}{|l|}{ mALBI grade } \\
\hline $1 / 2 \mathrm{a}(\mathrm{n}=248)$ & 1 & $1.083-3.037$ & 0.024 \\
\hline $2 \mathrm{~b} / 3(\mathrm{n}=29)$ & 1.814 & & \\
\hline \multicolumn{4}{|l|}{ BCLC stage } \\
\hline$\leq \mathrm{B}(\mathrm{n}=136)$ & 1 & $0.969-2.209$ & 0.070 \\
\hline$\geq C(\mathrm{n}=141)$ & 1.463 & & \\
\hline
\end{tabular}

Table 2. Multivariate analysis in patients with Child-Pugh score of 5. HR Hazard ratio, CI Confidence interval, ECOG-PS Eastern Cooperative Oncology Group performance status, HCC Hepatocellular carcinoma, $m A L B I$ Modified albumin-bilirubin, $B C L C$ Barcelona clinic liver cancer.

score for predictive overall survival at each month were nearly identical to the value that separated mALBI grades $2 \mathrm{a}$ and $2 \mathrm{~b}$. The optimal cut-off value of the Child-Pugh score for predictive overall survival was 5 at each month.

\section{Discussion}

In this multicenter study of a large number of patients with unresectable HCC who received lenvatinib therapy, Cox proportional hazards modeling that included age, sex, ECOG-PS, HCC etiology, mALBI grade, $\alpha$-fetoprotein, and BCLC stage as covariates showed that mALBI grade (1/2a vs. $2 \mathrm{~b} / 3)$ was independently associated with overall 


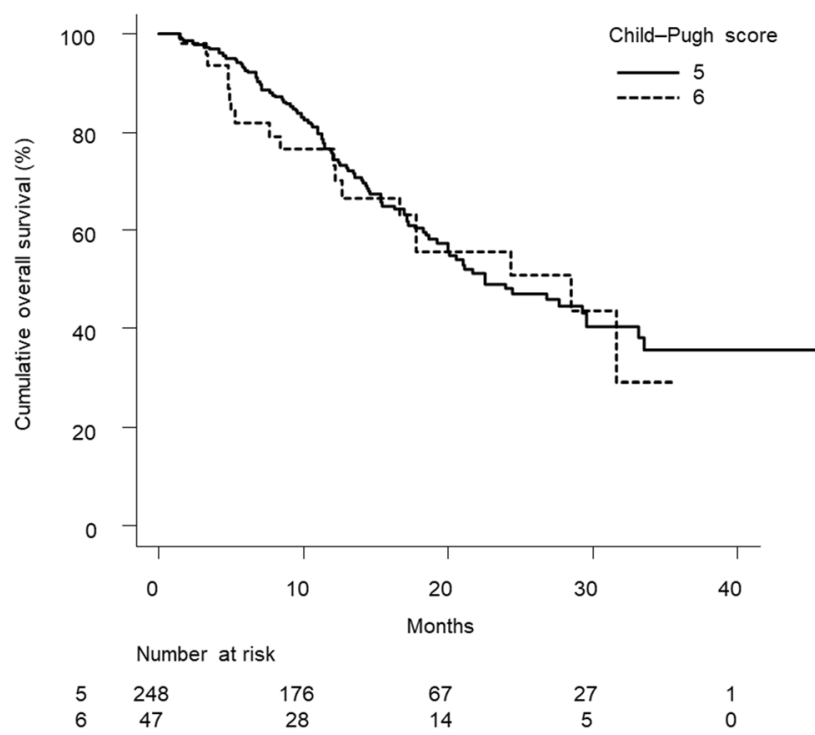

Figure 3. Cumulative survival curves in patients with mALBI grade $1 / 2 \mathrm{a}$ and Child-Pugh class A. There was no difference in cumulative overall survival between patients with mALBI grade $1 / 2 \mathrm{a}$ and Child-Pugh classes 5 and $6(p=0.735)$. The median survival times in patients with mALBI grade $1 / 2 \mathrm{a}$ and Child-Pugh classes 5 and 6 were 22.5 (95\%CI, 19.2-29.5) and 28.5 (95\%CI, 12.7-not available) months, respectively. $m A L B I$ Modified albumin-bilirubin.

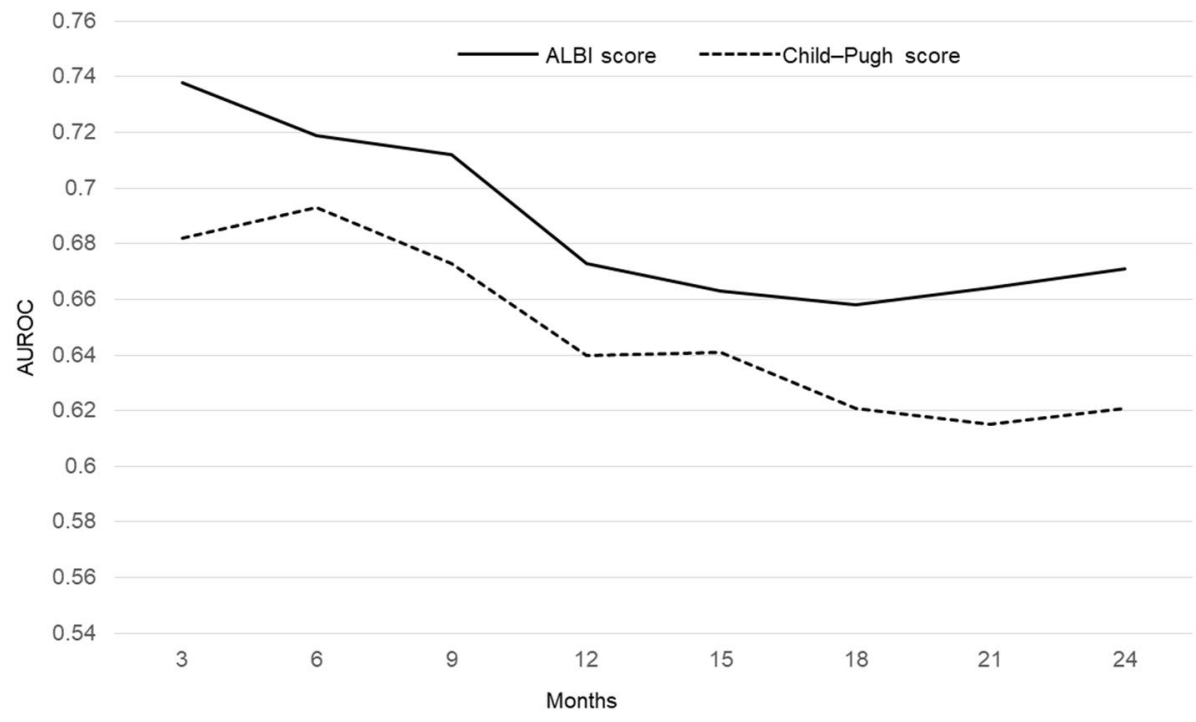

Figure 4. Time-dependent AUROCs of the ALBI and Child-Pugh scores for overall survival after the start of follow-up. Based on time-dependent ROC analysis, the AUROCs of the ALBI and Child-Pugh scores for overall survival at $3,6,9,12,15,18,21$, and 24 months were 0.738 and $0.682,0.719$ and $0.693,0.712$ and $0.673,0.673$ and $0.640,0.663$ and $0.641,0.658$ and $0.621,0.664$ and 0.615 , and 0.671 and 0.621 , respectively. Based on timedependent AUROCs, the ALBI score had a higher predictive power for overall survival than the Child-Pugh score. AUROC Area under the receiver operating characteristic curve, ALBI Albumin-bilirubin.

survival in patients with a Child-Pugh score of 5. Conversely, the survival analysis of patients with mALBI grade 1/2a and Child-Pugh class A showed no difference between patients with Child-Pugh class 5 versus 6 . In addition, the predictive power of the ALBI score for overall survival was superior to that of the Child-Pugh score in time-dependent ROC analysis. Furthermore, the optimal cut-off values of the ALBI score for predicting good survival in the 2 years from the start of lenvatinib treatment were nearly equal to the value separating $\mathrm{mALBI}$ grades $2 \mathrm{a}$ and $2 \mathrm{~b}$. These results suggest that the ALBI score is a better predictive marker for overall survival than the Child-Pugh score in patients with unresectable HCC who are treated with lenvatinib. In addition, the mALBI grade was able to predict good versus poor prognosis in patients with a Child-Pugh score of 5, even though these patients are generally considered to have a good prognosis. Furthermore, patients with a mALBI grade of 


\begin{tabular}{|l|l|l|l|l|l|l|}
\hline & \multicolumn{9}{|l|}{ ALBI score } & \multicolumn{3}{l|}{ Child-Pugh score } \\
\cline { 2 - 7 } & Cut-off value & Sensitivity (\%) & Specificity (\%) & Cut-off value & Sensitivity (\%) & Specificity (\%) \\
\hline At 6 months & -2.19 & 70.0 & 67.8 & 5 & 73.9 & 58.5 \\
\hline At 12 months & -2.24 & 61.3 & 71.9 & 5 & 63.1 & 62.8 \\
\hline At 18 months & -2.24 & 54.4 & 74.3 & 5 & 58.1 & 65.1 \\
\hline At 24 months & -2.31 & 56.8 & 73.9 & 5 & 55.2 & 66.6 \\
\hline
\end{tabular}

Table 3. Sensitivity, specificity, and cut-off values based on time-dependent ROC analysis. ROC Receiver operating characteristic.

1 or $2 \mathrm{a}$ had a similar prognosis regardless of whether their Child-Pugh score was 5 or 6 . In the present study, the median overall survival was longer than those of RFLECT trial ${ }^{10}$ (17.1 vs. 13.6 months). It was considered that the evolution of post-treatment of lenvatinib and the good management of adverse events were influenced the improvement of median overall survival.

Recently, Ueshima et al. ${ }^{22}$ investigated the association between baseline liver function, as determined by the Child-Pugh score and ALBI grade, and the outcomes of 82 patients with unresectable HCC who were treated with lenvatinib. Their study patients were divided into four groups: (1) Child-Pugh score 5 and ALBI grade 1 (group 1; $\mathrm{n}=27$ ); (2) Child-Pugh score 5 and ALBI grade 2 (group 2; $=19$ ); (3) Child-Pugh score 6 (group 3; $\mathrm{n}=30$ ); and (4) Child-Pugh score $\geq 7$ (group $4 ; \mathrm{n}=6$ ). They found that the median times to treatment failure (i.e., time from the initial administration of lenvatinib to treatment discontinuation for any reason, including disease progression, treatment toxicity, patient preference, and any cause of death) were 8.9, 5.3, 5.9 and 0.3 months in groups $1,2,3$, and 4 , respectively (HR, $0.38 ; 95 \% \mathrm{CI}, 0.18-0.80 ; p<0.001)^{22}$. In addition, overall survival was significantly better in patients with ALBI grade 1 than in those with ALBI grade 2 (HR, 0.12; 95\%CI, 0.02-0.97); $p<0.01)^{22}$. Very recently, Tsuchiya et al. ${ }^{23}$ investigated the factors associated with overall survival in 343 patients with unresectable HCC who were treated with lenvatinib. They found that ECOG-PS $\geq 1(\mathrm{HR}, 1.50 ; 95 \% \mathrm{CI}$, $1.09-2.08 ; p=0.014$ ), mALBI grade $2 \mathrm{~b} / 3$ (HR, 1.56; 95\%CI, 1.09-2.17; $p=0.012$ ), $a$-fetoprotein $\geq 400$ (HR, 2.00; $95 \% \mathrm{CI}, 1.42-2.80 ; p<0.001$ ), major vascular invasion (HR, 1.91; 95\%CI, 1.26-2.89; $p=0.002$ ), and molecular targeted therapy experience (HR, $2.22 ; 95 \% \mathrm{CI}, 1.56-3.13 ; p<0.001)$ were independently associated with overall survival in the multivariate analysis. Although our study did not investigate the time to treatment failure, we showed that patients with mALBI grade 1 , as well as those with $2 \mathrm{a}$, had better overall survival than patients with mALBI grade $2 \mathrm{~b} / 3$. In addition, we used time-dependent ROC analysis to demonstrate that the optimal cut-off values of the ALBI score during the 2 years after initiating lenvatinib treatment were nearly equal to the value that separated mALBI grades $2 \mathrm{a}$ and $2 \mathrm{~b}$, as opposed to that separating ALBI grades 1 and 2 . One advantage of this study relative to that by Ueshima et al. and Tsuchiya et al. is that the former included more patients with unresectable HCC who received lenvatinib therapy. In addition, this study statistically confirmed the optimal cut-off values of the ALBI score for predicting good overall survival in patients treated with lenvatinib.

Ando et al. ${ }^{24}$ reported that $\mathrm{mALBI}$ grade $1 / 2 \mathrm{a}$ (odds ratio, 5.18 ; 95\%CI, $1.465-18.31 ; p=0.011$ ) was an independent factor for possible treatment with second-line molecularly targeted agents in $141 \mathrm{HCC}$ patients who received lenvatinib as first-line therapy. In addition, in a study by Hiraoka et al. ${ }^{25}$ using a Japanese hospitalbased administration database, the overall duration of systematic treatment in patients with advanced HCC was shorter in those with a baseline ALBI grade of $2 \mathrm{~b}$ or 3 than in those with grade 1 or $2 \mathrm{a}$ (medians: 7.1, 6.7, 4.5, and 3.0 months for grades $1,2 \mathrm{a}, 2 \mathrm{~b}$, and 3 , respectively). In this study, we clarified that there was a significant difference in the post-treatment of lenvatinib rate between patients with $\mathrm{mALBI}$ grade $1 / 2 \mathrm{a}$ and those with grade $2 \mathrm{~b} / 3$, even among those with good liver function as defined by a Child-Pugh score of 5 .

It is well known that immunology regulations may affect HCC progression and it was reported that immunodeficiency may promote adaptive alterations of host gut- or tissue-based microbiome ${ }^{26}$. Therefore, it was considered that these phenomena may be influenced mALBI grade as potential mechanisms and associated with survival in patients with HCC who received lenvatinib.

The mRNA modifications are potentially new insights into this biological basis, especially N4-acetylcytidine on RNA expression ${ }^{27}$. The recent progress in N4-acetylcytidine on RNA expression is also playing key role on the development of HCC and survival in patients with HCC. Further prospective studies with considering the relationship between mALBI grade and mRNA modifications/ N4-acetylcytidine, including additional potential mechanisms, in patients with HCC are warranted.

The Child-Pugh score/classification system comprises five factors, specifically serum albumin, total bilirubin, prothrombin time, ascites, and encephalopathy ${ }^{14}$. This system has been widely used to evaluate hepatic function and has been incorporated into the HCC staging system ${ }^{28}$. However, the Child-Pugh score is limited by the subjectivity involved in evaluating hepatic encephalopathy and ascites, and serum albumin levels are associated with the severity of ascites ${ }^{14}$. Furthermore, this hepatic function assessment system was originally developed for patients with cirrhosis, not HCC. The ALBI grade, which was recently developed as an objective biomarker for assessment of hepatic function, is calculated using only serum albumin and total bilirubin levels. It has been shown to accurately predict the prognosis of patients with HCC, and is superior not only to the Child-Pugh classification $^{20,29}$, but also to the liver damage classification system $^{30}$.

Hiraoka et al. ${ }^{16}$ developed the new mALBI grading system, which divides ALBI grade 2 into $2 \mathrm{a}$ and $2 \mathrm{~b}$, by analyzing 46,681 HCC patients in a nationwide survey conducted in Japan. The mALBI grade showed a good ability to stratify prognosis in each TNM stage of the Liver Cancer Study Group of Japan ${ }^{31}$, and there was a 
statistically significant difference between each mALBI grade in all TMN stages $(p<0.01)$. This study confirmed the utility of subdividing ALBI grade 2 into $2 \mathrm{a}$ and $2 \mathrm{~b}$ for predicting survival in advanced HCC patients who received systemic therapy.

ROC analysis is generally used to assess the discriminatory ability of a continuous variable for a binary disease outcome (e.g., disease positive or negative). However, majority of chronic disease outcomes, including the prognosis of patients with cancer, are time dependent. Therefore, time-dependent ROC curve analysis has been used to assess the predictive ability of clinical markers for time-dependent disease outcomes ${ }^{21}$. No previous studies have used this statistical method to assess parameters of hepatic function regarding to their association with overall survival in patients with advanced HCC who treated with lenvatinib. In this study, the AUROCs by time-dependent ROC curve analysis showed that the ALBI score was superior to the Child-Pugh score in terms of predicting overall survival up to 2 years after the start of lenvatinib therapy in patients with unresectable HCC.

The main limitations of this study are its hospital-based subject population and retrospective nature. Although the study included a large number of patients with unresectable HCC from multiple centers in Japan, future prospective studies with community-based populations are warranted. An additional limitation was that the study only enrolled patients with unresectable HCC who were treated with lenvatinib. Future studies of patients with HCC should include those who receive atezolizumab plus bevacizumab, a recently approved, first-line, systemic combination therapy, as well as those who receive lenvatinib.

In conclusion, the mALBI grade is a new, simple, objective parameter that was a better predictor of survival in HCC patients who received lenvatinib therapy than the Child-Pugh classification, even among individuals with good liver function as defined by a Child-Pugh score of 5. Further studies in other populations are warranted to confirm these findings.

\section{Methods}

Patients. The protocol used in the present study was approved by the Institutional Ethics Committee of Ehime Prefectural Central Hospital (IRB No. 30-66), based on the Guidelines for Clinical Research issued by the Ministry of Health and Welfare of Japan. All methods were carried out in accordance with relevant guidelines and regulations.

We enrolled 524 patients with unresectable HCC who received lenvatinib between March 2018 and February 2021 at 19 institutions in Japan [Himeji Red Cross Hospital $(n=126)$, Nippon Medical School Hospital Group (Sendagi Hospital, Chiba Hokusoh Hospital, and Musashi Kosugi Hospital) ( $n=75)$, Ehime Prefectural Central Hospital ( $n=53)$, Ogaki Municipal Hospital $(n=38)$, Kagawa University Hospital $(n=33)$, Asahi General Hospital $(n=26)$, Ehime University Hospital $(n=23)$, Osaka Medical College Hospital $(n=22)$, Okayama City Hospital $(n=21)$, Teine Keijinkai Hospital $(n=20)$, Saiseikai Niigata Hospital $(n=18)$, Kagawa Prefectural Central Hospital $(n=17)$, Hamamatsu University Hospital $(n=17)$, Matsuyama Red Cross Hospital $(n=16)$, Otakanomori Hospital $(n=9)$, Toyama University Hospital $(n=6)$, and Tokushima Prefectural Central Hospital $(n=4)]$.

The start of the follow-up period was defined as the date when lenvatinib therapy began. The end of followup was defined as the date of the final visit for patients who remained alive or the date of death for patients who died during the follow-up period.

The etiology of HCC was considered to be hepatitis B virus in patients positive for hepatitis B virus surface antigen, and hepatitis $\mathrm{C}$ virus in those positive for hepatitis $\mathrm{C}$ virus antibodies.

Diagnosis and treatment of HCC. HCC was diagnosed according to increases in a-fetoprotein levels, pathological findings, or the use of imaging modalities such as dynamic computed tomography, gadolinium ethoxybenzyl diethylenetriamine pentaacetic acid-enhanced magnetic resonance imaging, and contrast-enhanced ultrasonography with perflubutane ${ }^{32,33}$. HCC stage was based on the Barcelona Clinic Liver Cancer (BCLC) classification system ${ }^{28}$.

The most appropriate treatment modality for HCC in each patient was selected through discussion between surgeons, hepatologists, and radiologists in each institution, according to Japanese practice guidelines for $\mathrm{HCC}^{34,35}$.

Liver function assessment. We assessed liver function using the Child-Pugh classification system ${ }^{14}$ and albumin-bilirubin (ALBI) score ${ }^{15}$. For more detailed evaluation of hepatic function, we used the mALBI grade, which was calculated by subdividing ALBI grade 2 into $2 \mathrm{a}$ and $2 \mathrm{~b}^{16}$.

Lenvatinib treatment. Lenvatinib (LENVIMA; Eisai, Tokyo, Japan) treatment was started after written informed consent was obtained from each patient. The dose of oral lenvatinib was $8 \mathrm{mg} /$ day in patients who weighed $<60 \mathrm{~kg}$ and $12 \mathrm{mg} /$ day in those who weighed $\geq 60 \mathrm{~kg}$. However, the initial dose of lenvatinib was reduced at the discretion of the physician in patients with any of the following: non-Child-Pugh A disease; advanced age; low body weight; pleural effusion, ascites, or gastrointestinal varices at risk of bleeding.

Lenvatinib was discontinued when any unacceptable or serious adverse event or clinical tumor progression occurred. According to the drug manufacturer's guidelines, the lenvatinib dose was reduced or treatment was interrupted when a patient occurred any grade $\geq 3^{36}$ severe adverse events, if any intolerable treatment-related adverse events developed, or if there was clinical evidence of HCC progression. In cases of treatment-related adverse events, dosing was reduced or temporarily interrupted until symptoms diminished to grade 1 or 2 , according to the manufacturer's guidelines.

Evaluation of therapeutic response. Local physicians at each institution evaluated tumors using enhanced computed tomography or magnetic resonance imaging results obtained at 4 or 12 weeks after intro- 
ducing lenvatinib, in accordance with the modified Response Evaluation Criteria in Solid Tumors (RECIST) guidelines $^{37,38}$.

Statistical analysis. Continuous variables are expressed as medians (interquartile range). The chi-square test was used for categorical variables. Actuarial analysis of cumulative survival was performed using the Kaplan-Meier method, and differences were tested using the log-rank test with Bonferroni correction. Univariate and multivariate Cox proportional hazards models were used to calculate hazard ratios (HRs) for survival. The concordance $(\mathrm{C})$-index was used to determine the predictability of survival. We performed multivariate analysis using the following covariates that were previously reported to be risk factors for HCC or predictors of hepatic prognosis: age, sex, Eastern Cooperative Oncology Group performance status (ECOG-PS), HCC etiology, a-fetoprotein, HCC stage, and mALBI grade ${ }^{4,13,39,40}$. We used cut-off values for clinical data as defined in previous reports regarding the risk or prognosis of patients with $\mathrm{HCC}^{4,13,39,40}$. In this study, we analyzed clinical data obtained at the start of follow-up. Time-dependent ROC curves for overall survival were obtained with the Kaplan-Meier method using the Child-Pugh score and ALBI score. We calculated the sensitivity and specificity at each survival time using the maximum Youden index (sensitivity + specificity -1 ) as the cut-off value ${ }^{41,42}$.

Statistical significance was defined as $p<0.05$. Statistical analyses were performed with EZR Ver. 1.53 (Saitama Medical Center, Jichi Medical University, Saitama, Japan), which is a graphical user interface for R (The R Foundation for Statistical Computing, Vienna, Austria $)^{43}$. More precisely, it is a modified version of the $\mathrm{R}$ commander designed to add statistical functions frequently used in biostatistics.

Ethics approval. The protocol used in the present study was approved by the Institutional Ethics Committee of Ehime Prefectural Central Hospital (IRB No. 30-66), based on the Guidelines for Clinical Research issued by the Ministry of Health and Welfare of Japan. All methods were carried out in accordance with relevant guidelines and regulations.

Consent to participate. Written informed consent was obtained from each patient.

\section{Data availability}

The datasets are available from the corresponding author on reasonable request.

Received: 1 June 2021; Accepted: 30 June 2021

Published online: 14 July 2021

\section{References}

1. Forner, A., Reig, M. \& Bruix, J. Hepatocellular carcinoma. Lancet 391, 1301-1314 (2018).

2. Kim, D. Y. \& Han, K. H. Epidemiology and surveillance of hepatocellular carcinoma. Liver Cancer 1, 2-14 (2012).

3. Nault, J. C., Galle, P. R. \& Marquardt, J. U. The role of molecular enrichment on future therapies in hepatocellular carcinoma. J. Hepatol. 69, 237-247 (2018).

4. European Association for the Study of the Liver; European Organisation for Research and Treatment of Cancer. EASL-EORTC clinical practice guidelines: Management of hepatocellular carcinoma. J. Hepatol. 56, 908-943 (2012).

5. Llovet, J. M. et al. SHARP Investigators Study Group. Sorafenib in advanced hepatocellular carcinoma. N. Engl. J. Med. 359, 378-390 (2008).

6. Cheng, A. L. et al. Safety and efficacy of sorafenib in patients in the Asia-Pacific region with advanced hepatocellular carcinoma: A phase III randomised, double-blind, placebo-controlled trial. Lancet Oncol. 10, 25-34 (2009).

7. Bruix, J. et al. RESORCE Investigators. Regorafenib for patients with hepatocellular carcinoma who progressed on sorafenib treatment (RESORCE): A randomised, double-blind, placebo-controlled, phase 3 trial. Lancet 389, 56-66 (2017).

8. Finn, R. S. et al. Outcomes of sequential treatment with sorafenib followed by regorafenib for HCC: Additional analyses from the phase III RESORCE trial. J. Hepatol. 69, 353-358 (2018).

9. Zhu, A. X. et al. REACH-2 study investigators. Ramucirumab after sorafenib in patients with advanced hepatocellular carcinoma and increased $\alpha$-fetoprotein concentrations (REACH-2): A randomised, double-blind, placebo-controlled, phase 3 trial. Lancet Oncol. 20, 282-296 (2019).

10. Kudo, M. et al. Lenvatinib versus sorafenib in first-line treatment of patients with unresectable hepatocellular carcinoma: A randomised phase 3 non-inferiority trial. Lancet 391, 1163-1173 (2018).

11. Hiraoka, A. et al. Real-life Practice Experts for HCC (RELPEC) Study Group and the HCC 48 Group (hepatocellular carcinoma experts from 48 clinics in Japan). Therapeutic potential of lenvatinib for unresectable hepatocellular carcinoma in clinical practice: Multicenter analysis. Hepatol. Res. 49, 111-117 (2019).

12. Hiraoka, A. et al. Real-life Practice Experts for HCC (RELPEC) Study Group, HCC 48 Group (hepatocellular carcinoma experts from 48 clinics in Japan); GLSG (Gunma Liver Study Group). Therapeutic efficacy of lenvatinib as third line treatment following regorafenib for unresectable hepatocellular carcinoma progression. Hepatol. Res. 2021 Apr 10. https://doi.org/10.1111/hepr.13644. Online ahead of print.

13. Finn, R. S. et al. IMbrave150 Investigators. Atezolizumab plus Bevacizumab in Unresectable Hepatocellular Carcinoma. N. Engl. J. Med. 382, 1894-1905 (2020).

14. Pugh, R. N., Murray-Lyon, I. M., Dawson, J. L., Pietroni, M. C. \& Williams, R. Transection of the oesophagus for bleeding oesophageal varices. Br. J. Surg. 60, 646-649 (1973).

15. Johnson, P. J. et al. Assessment of liver function in patients with hepatocellular carcinoma: A new evidence-based approach-the ALBI grade. J. Clin. Oncol. 33, 550-558 (2015).

16. Hiraoka, A. et al. Validation and potential of albumin-bilirubin grade and prognostication in a nationwide survey of 46,681 hepatocellular carcinoma patients in Japan: The need for a more detailed evaluation of hepatic function. Liver Cancer. 6, 325-336 (2017).

17. Hatanaka, T. et al. The role of the albumin-bilirubin score for predicting the outcomes in Japanese patients with advanced hepatocellular carcinoma treated with ramucirumab: A real-world study. Oncology 99, 203-214 (2021).

18. Chen, H. Y. et al. Predicting outcomes for recurrent hepatocellular carcinoma within Milan criteria after complete radiofrequency ablation. PLoS ONE 15, e0242113 (2020). 
19. Kumada, T., Toyoda, H., Tada, T., Yasuda, S. \& Tanaka, J. Changes in background liver function in patients with hepatocellular carcinoma over 30 years: Comparison of Child-Pugh classification and albumin bilirubin grade. Liver Cancer 9, 518-528 (2020).

20. Tada, T. et al. Impact of albumin-bilirubin grade on survival in patients with hepatocellular carcinoma who received sorafenib: An analysis using time-dependent receiver operating characteristic. J. Gastroenterol. Hepatol. 34, 1066-1073 (2019).

21. Heagerty, P. J. \& Zheng, Y. Survival model predictive accuracy and ROC curves. Biometrics 61, 92-105 (2005).

22. Ueshima, K. et al. Impact of baseline ALBI grade on the outcomes of hepatocellular carcinoma patients treated with lenvatinib: A multicenter study. Cancers (Basel) 11, 952 (2019).

23. Tsuchiya, K. et al. The real-world data in Japanese patients with unresectable hepatocellular carcinoma treated with lenvatinib from a nationwide multicenter study. Cancers (Basel) 13, 2608 (2021).

24. Ando, Y. et al. Analysis of post-progression survival in patients with unresectable hepatocellular carcinoma treated with lenvatinib. Oncology 98, 787-797 (2020).

25. Hiraoka, A., Tanizawa, Y., Huang, Y. J., Cai, Z. \& Sakaguchi S. Association of albumin-bilirubin grade and sequential treatment with standard systemic therapies for advanced hepatocellular carcinoma: A retrospective cohort study using a Japanese administrative database. Drugs Real World Outcomes. 2021 Apr 1. https://doi.org/10.1007/s40801-021-00245-8. Online ahead of print.

26. Zheng, S. et al. Immunodeficiency promotes adaptive alterations of host gut microbiome: An observational metagenomic study in mice. Front. Microbiol. 10, 2415 (2019).

27. Jin, G. et al. The processing, gene regulation, biological functions, and clinical relevance of N4-acetylcytidine on RNA: A systematic review. Mol. Ther. Nucl. Acids 20, 13-24 (2020).

28. Llovet, J. M., Brú, C. \& Bruix, J. Prognosis of hepatocellular carcinoma: The BCLC staging classification. Semin. Liver Dis. 19, 329-338 (1999).

29. Marrero, J. A. et al. Diagnosis, staging, and management of hepatocellular carcinoma: 2018 Practice guidance by the American Association for the study of liver diseases. Hepatology 68, 723-750 (2018).

30. Hiraoka, A. et al. Real-Life Practice Experts for HCC (RELPEC) Study Group and HCC 48 Group (hepatocellular carcinoma experts from 48 clinics). Albumin-bilirubin (ALBI) grade as part of the evidence-based clinical practice guideline for HCC of the Japan Society of hepatology: A comparison with the liver damage and Child-Pugh classifications. Liver Cancer 6, 204-215 (2017).

31. Liver Cancer Study Group of Japan: The General Rules for the Clinical and Pathological Study of Primary Liver Cancer, 6th edn. Tokyo, Kanehara (2015).

32. Bruix, J. \& Sherman, M. Management of hepatocellular carcinoma. Hepatology 42, 1208-1236 (2005).

33. Bruix, J. \& Sherman, M. Management of hepatocellular carcinoma: An update. Hepatology 53, 1020-1022 (2011).

34. Kudo, M. et al. Liver Cancer Study Group of Japan. JSH Consensus-Based Clinical Practice Guidelines for the Management of Hepatocellular Carcinoma: 2014 Update by the Liver Cancer Study Group of Japan. Liver Cancer. 3, 458-468 (2014).

35. Kokudo, N. et al. Evidence-based clinical practice guidelines for hepatocellular carcinoma: The Japan Society of Hepatology 2013 update (3rd JSH-HCC guidelines). Hepatol. Res. https://doi.org/10.1111/hepr.12464 (2015).

36. National Cancer Institute, Protocol Development Cancer Therapy. https://ctep.cancer.gov/protocolDevelopment/electronic_appli cations/ctc.htm-ctc_40. Accessed 1 April 2021.

37. Eisenhauer, E. A. et al. New response evaluation criteria in solid tumours: Revised RECIST guideline (version 1.1). Eur J Cancer. 45, 228-247 (2009).

38. Lencioni, R. \& Llovet, J. M. Modified RECIST (mRECIST) assessment for hepatocellular carcinoma. Semin. Liver Dis. 30, 52-60 (2010).

39. Kaibori, M. et al. Liver Cancer Study Group of Japan. Impact of advanced age on survival in patients undergoing resection of hepatocellular carcinoma: Report of a Japanese Nationwide Survey. Ann. Surg. 269, 692-699 (2019).

40. Tada, T. et al. Neutrophil-to-lymphocyte ratio is associated with survival in patients with unresectable hepatocellular carcinoma treated with lenvatinib. Liver Int. 40, 968-976 (2020).

41. Youden, W. J. Index for rating diagnostic tests. Cancer 3, 32-35 (1950).

42. Yu, H. et al. LEPR hypomethylation is significantly associated with gastric cancer in males. Exp. Mol. Pathol. 116, 104493 (2020).

43. Kanda, Y. Investigation of the freely available easy-to-use software 'EZR' for medical statistics. Bone Marrow Transpl. 48, 452-458 (2013).

\section{Author contributions}

Concept and study design: A.H. and T.T., Data acquisition: All authors, Analyses of the data: A.H. and T.T., Statistics: T.T., Supervise: T.K., Preparing manuscript: T.T., Review and approval: All authors.

\section{Funding}

There was no Grant support or other financial support for this study.

\section{Competing interests}

The authors declare no competing interests.

\section{Additional information}

Supplementary Information The online version contains supplementary material available at https://doi.org/ 10.1038/s41598-021-93794-5.

Correspondence and requests for materials should be addressed to T.T.

Reprints and permissions information is available at www.nature.com/reprints.

Publisher's note Springer Nature remains neutral with regard to jurisdictional claims in published maps and institutional affiliations. 
(c) (i) Open Access This article is licensed under a Creative Commons Attribution 4.0 International cc) License, which permits use, sharing, adaptation, distribution and reproduction in any medium or format, as long as you give appropriate credit to the original author(s) and the source, provide a link to the Creative Commons licence, and indicate if changes were made. The images or other third party material in this article are included in the article's Creative Commons licence, unless indicated otherwise in a credit line to the material. If material is not included in the article's Creative Commons licence and your intended use is not permitted by statutory regulation or exceeds the permitted use, you will need to obtain permission directly from the copyright holder. To view a copy of this licence, visit http://creativecommons.org/licenses/by/4.0/.

(C) The Author(s) 2021 